\title{
Parent-Child Interaction Therapy (PCIT) for young children with Attention-Deficit Hyperactivity Disorder (ADHD) in Japan
}

\author{
Nana Hosogane ${ }^{1 *}\left(\mathbb{0}\right.$, Masaki Kodaira ${ }^{1}$, Nozomi Kihara ${ }^{2}$, Kazuhiko Saito ${ }^{3}$ and Toshiko Kamo ${ }^{4}$
}

\begin{abstract}
Early intervention for preschoolers with Attention-Deficit Hyperactivity Disorder (ADHD) is important considering the impact on their prognosis. Parent-Child Interaction Therapy (PCIT) is a psychotherapy treatment for the parent-child dyad and has been shown to be effective for children with disruptive behaviors. We present the treatment course of PCIT for two Japanese children with ADHD. Case 1 is a 2-year-old female child with hyperactivity and aggressiveness. Case 2 is a 4-year-old male child with restlessness and intolerability to daily events. For both cases, PCIT was effective in improving the problematic behaviors. PCIT may serve as a treatment option for Japanese children with ADHD.
\end{abstract}

Keywords: Attention-Deficit Hyperactivity Disorder (ADHD), Treatment, Parent-Child Interaction Therapy (PCIT), Preschooler

\section{Background}

Young children with Attention-Deficit Hyperactivity Disorder (ADHD) are often referred to services for their disruptive behaviors, such as difficulty in obeying commands in pre-school/day-care, tantrums or aggressiveness; all which influence relationships with others. Early interventions for these preschoolers are important taking into account of their prognosis that may lead to impairments in academic, interpersonal, and occupational domains. Treatment guidelines for children with ADHD recommend environmental adjustments, psychosocial treatment, and psychopharmacotherapy according to the severity of impairment [1]. Psychostimulant medications which are regarded as first-line treatment in psychopharmacotherapy are not recommended for children under age of 6 because of the paucity of investigation of the efficacy and safety in this age group [2]. Parent behavior training (PBT) interventions have greater evidence of effectiveness for preschoolers with ADHD [3]. PBT interventions refer to interventions in which parents learn

\footnotetext{
*Correspondence: nhosogane@hotmail.com

${ }^{1}$ Department of Child and Adolescent Mental Health, Aiiku Clinic, Maternal and Child Health Center, Imperial Gift Foundation Boshi-AiikuKai, 5-6-8 Minami-Azabu, Minato-ku, Tokyo 106-8580, Japan

Full list of author information is available at the end of the article
}

certain skills to improve children's problematic behavior, such as group-based parent-training $[4,5]$, Positive Parenting Program (Triple P) [6], or Parent-Child Interaction Therapy (PCIT) [7].

PCIT is an individualized psychotherapy treatment for the child-parent dyad based on behavioral therapy. It has been developed to improve externalizing problematic behavior through the improvement of child-parent interaction. Meta-analysis has shown that PCIT is effective for young children with disruptive behavior [8] and efficacy has been shown to be maintained for 3-6 years after treatment [9].

PCIT is a manualized treatment with core defining features. It consists of two phases: Child-Directed Interaction (CDI) and Parent-Directed Interaction (PDI). PCIT skills are taught didactically to parents in the first session of each phase ("teaching session") and also through direct coaching in the following sessions ("coaching session") where the therapists watch the dyad play in the play-room behind the one-way mirror and coaches directly to the parent using a microphone and "bug-inthe-ear" device. In this way, the therapists are able to give immediate feedback to the parent and reinforce behavior management skills while interacting with the child. The parent can also validate how certain skills are effective for 
their child. Treatment proceeds according to the parent's skills and improvement of the child. PCIT often consists of 12-20 weekly sessions.

During the first phase (CDI), parents are taught positive parenting skills while they are following the child's lead during their interaction. These skills are called "PRIDE skills": Praise, Reflect, Imitate, Describe, and Enjoy (Enthusiasm). They are also taught to avoid negative interactions: criticism, questions, and commands, which take the lead away from the child, and also to ignore inappropriate behavior. Parents are assessed at the beginning of each coaching session for $5 \mathrm{~min}$ and when parents meet mastery criteria, which is pre-set in the manual, they then move on to the next phase.

During the second phase (PDI), parents learn to give effective commands and to follow the protocol for consequence according to the child's behavior: compliance or non-compliance. When parents meet the mastery criteria for PDI skills and child's behavior reach the normal range (according to a behavior checklist (Eyberg Child Behavior Inventory; ECBI)), PCIT reaches completion. Although the number of sessions differs according to the progress, the manual includes the essential components in each session and allows the family to acquire all necessary skills during treatment.

During both phases, daily homework is given to the parents to practice skills during interaction. Parents are gradually encouraged to use these skills not only during the 5-min interaction, but also during daily life.

PCIT has been developed in the USA by Sheila Eyberg and has been translated to several languages and disseminated to countries world-wide such as Australia, Germany, Norway, and Asian countries. In Japan, PCIT was introduced in 2005 and has been shown to be efficacious for children and parents who experienced domestic violence (DV) [10].

In this current paper, we describe the treatment course of PCIT for two young Japanese children diagnosed with ADHD. The first author served as the main therapist; she has completed training and was certified as a PCIT Therapist by PCIT International. This is the first article written in English to date describing the treatment of PCIT for Japanese ADHD children.

\section{Case presentation}

\section{Case 1}

"Anna Oka," a Japanese female child, was 2 years and 10 months old with no siblings. She was brought by her parents for her hyperactivity and aggressiveness. Anna was aggressive toward her parents and other children: often hitting, biting, and scratching them. She had problems with daily routines such as getting dressed and brushing her teeth. Her parents needed to either threaten her or hold her down to make her obey.

Her developmental history revealed that she had no physical illnesses or injuries and had no delay in developmental milestones including her intellectual development. She had no history of child abuse or any other types of negative life events that may explain her symptoms. Anna was diagnosed as Attention-Deficit Hyperactivity Disorder (ADHD)-Combined Type after psychiatric evaluation. Her psychiatrist performed an interview and observation about the ADHD symptoms according to the DSM-5 diagnostic criteria [11]. The presence of impairment was observable both at home and at her day-care center. She did not fulfill the diagnostic criteria for Autism Spectrum Disorder. Mr. Oka was a self-employed business owner and Ms. Oka worked part-time. Although parents showed some inconsistency in their parenting, that was not enough to explain her destructiveness.

Parenting skills were taught during regular outpatient clinic visits; however, Anna's parents still had difficulty controlling her temper. Medication was not recommended for Anna because of the paucity of evidence in this age group and also because the varieties of types of stimulants are limited in Japan. Parent-Child Interaction Therapy (PCIT) was recommended and weekly sessions started with Anna and her mother when she was 3 years and 1 month old.

PCIT sessions were held every week for $60-90 \mathrm{~min}$ using the Japanese version of the PCIT 2011 protocol [12] by two PCIT therapists (NH and NK). Outpatient visits were continued in a regular basis by her psychiatrist (MK).

Problematic behaviors were assessed using the parentscored Eyberg Child Behavior Inventory (ECBI; 13). The Japanese average intensity score is $100.1(\mathrm{SD}=24.6)$ and the average problem score is $6.57(\mathrm{SD}=6.46)$. At baseline, Anna scored 178 on her ECBI intensity scale and 28 on her ECBI problem scale which meant that she had high frequency and number of problems in disruptive behaviors.

Baseline interactions were assessed using the Dyadic Parent-Child Interaction Coding System (DPICS; 14). There are three play situations for assessment: Child-Led Play (CLP; following the child's lead), Parent-Led Play (PLP; leading the child), and Clean-Up (CU; cleaning up toys). During the three situations, Ms. Oka used many questions and critical statements that were thought to promote negative interactions. Regarding statements that promote positive interactions ("PRIDE skills"), she did not use any behavioral descriptions nor labeled praises and used only few reflective statements. Many commands were given to Anna during PLP and CU, but her compliance was low $(0 / 10=0 \%$ in PLP, $0 / 6=0 \%$ in CU). 
The atmosphere of the mother and child seemed enjoyable; however, Anna and Ms. Oka were slightly physically distanced.

During the first phase of PCIT, called CDI (childdirected interaction), Ms. Oka learned to follow Anna's lead and to use PRIDE skills whenever Anna was playing appropriately and to ignore her problematic behavior until it had diminished. Ms. Oka and Anna had high attendance and completed homework every week. As Ms. Oka learned to use her skills, she was able to praise appropriate behaviors that were opposite of Anna's problematic behaviors (e.g., "Thank you for speaking with a soft voice." "You're such a good girl sitting while playing."). This was effective in preventing Anna behaving disruptively. Ms. Oka tried to maintain her consistency so that Anna could understand what her mother was expecting from her. Anna's father sometimes joined the session and watched the dyad from the back of the oneway mirror with the therapists.

During the second phase of PCIT, called PDI (parentdirected interaction), Ms. Oka gave simple commands and followed the protocol according to Anna's response (i.e., obey or disobey). As the treatment progressed, Anna was able to listen to her mother's commands during session and this change was also seen at home. Although Anna was still very hyperactive, her aggressiveness had decreased and parents felt confident in their parenting as she was able to follow most commands in every-day life.

PCIT sessions consisted of 21 CDI sessions and 9 PDI sessions, total of 30 sessions until Ms. Oka had met the "mastery criteria" and Anna's disruptive behaviors were within normal limits. Ms. Oka mentioned that Anna was not as hyperactive and desired to stay with her more frequently.

Post-treatment evaluation showed that the ECBI scores were 68 on her intensity scale and 11 on her problem scale; both well within normal limits (Fig. 1). At the posttreatment evaluation, Ms. Oka used statements promoting positive interaction (PRIDE skills) more often than pre-treatment (Fig. 2). Ms. Oka gave direct commands when necessary and Anna's compliance had improved $(4 / 5=80 \%$ in PLP, $10 / 12=83 \%$ in CU; Fig. 3$)$. On the Parenting Stress Index-Short Form (PSI-SF; 15), her total stress score had decreased revealing that the therapy was also meaningful for Ms. Oka herself (Fig. 4).

Follow-up sessions were held and Anna's ECBI scores were 76 on her intensity scale and 18 on her problem scale 3 months post-treatment (Fig. 1). Problem score had increased but her intensity score was well within normal limits.

All figures show raw scores and data were not statistically analyzed.

\section{Case 2}

"Kou Kawa," a Japanese male child, was 4 years and 2 months old when he was brought by his mother to the Clinic for his restlessness and intolerability to daily events. His parents both worked full-time and he had no siblings. He did not have any history of physical illnesses or injuries and has no delay in verbal or physical developments. He had problems at pre-school where he easily got excited and had difficulty staying seated. He

\section{ECBI intensity scores}

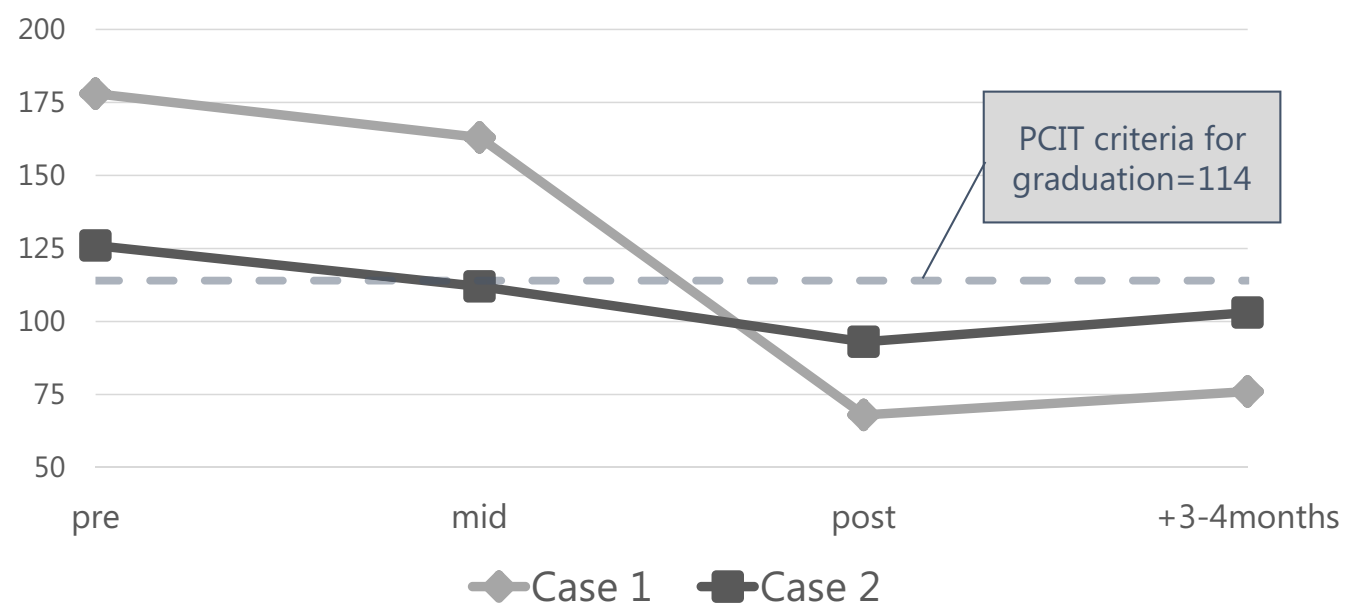

Fig. 1 Changes in problematic behavior according to ECBI intensity scores pre-, mid-, and post-treatment for Case 1 and Case 2. In both cases, the ECBI intensity scores were below the PCIT graduation criteria score $(<114)$ at post-treatment and follow-up (3-4 months after treatment). ECBI: Eyberg Child Behavior Inventory 
Case 1

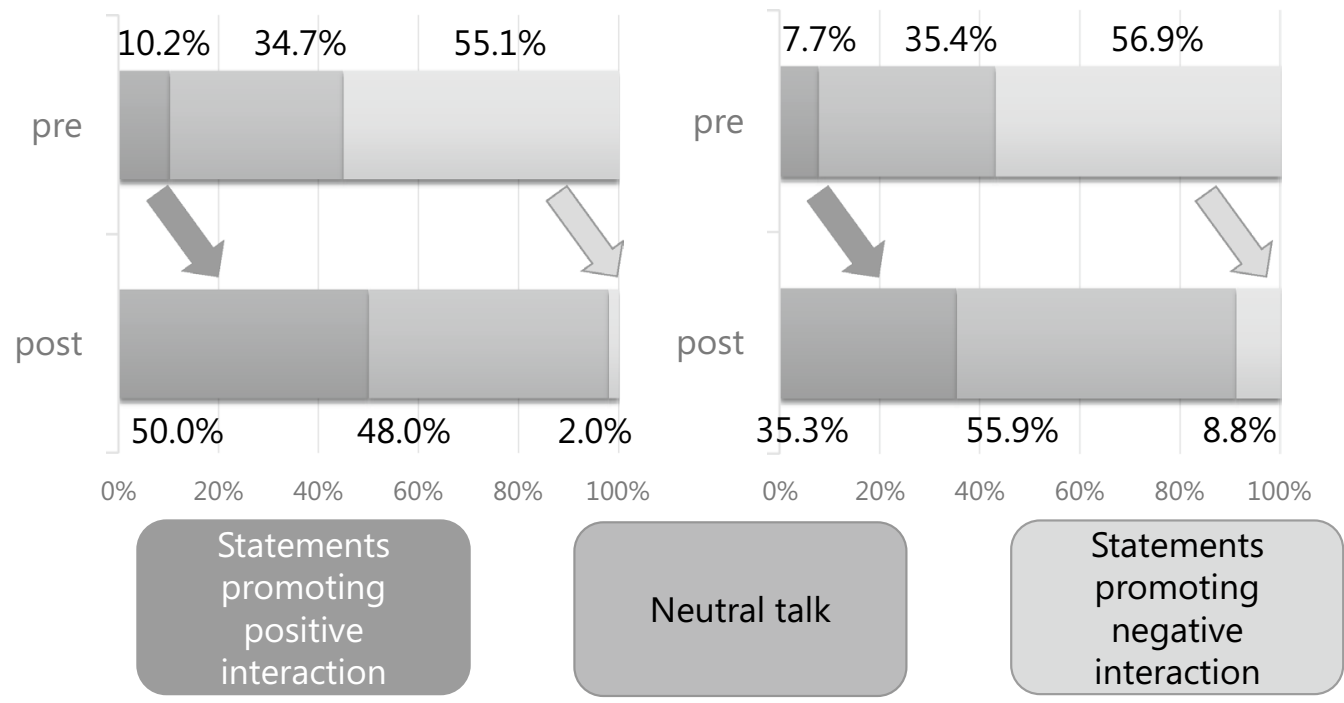

Fig. 2 Changes in quality of verbalization of parent in Case 1 and Case 2. The parents' verbalization was scored using the DPICS-IV during 5 min of CLP in pre- and post-treatment. The percentage of the quality of verbalization is shown. Statements were classified into three groups: statements promoting positive interaction (labeled and unlabeled praises, reflections, and descriptions), statements promoting negative interaction (questions, criticisms, commands), and neutral talk (other statements). Both mothers showed improvement in using statements that promoted positive interactions. DPICS-IV: Dyadic Parent-Child Interaction Coding System (4th edition), CLP: Child-Led Play

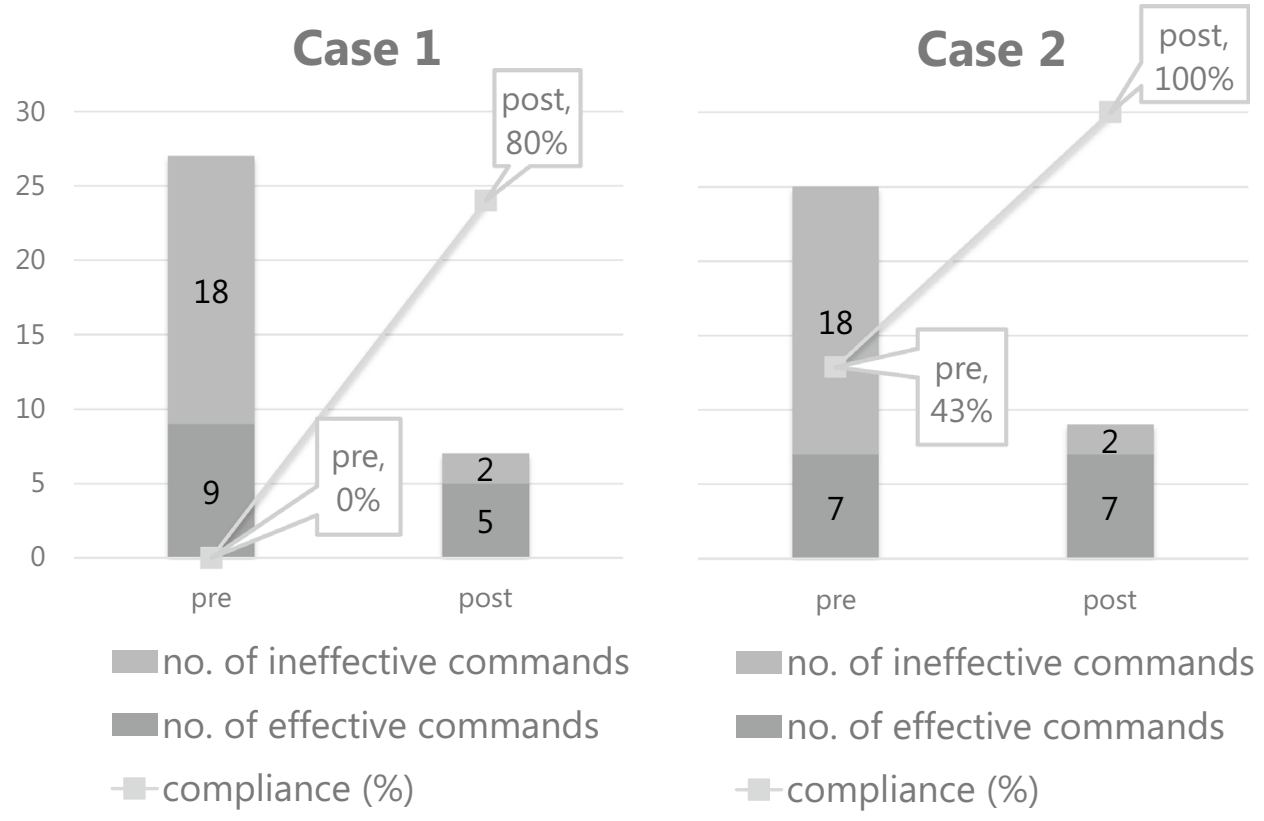

Fig. 3 Changes in numbers of commands and compliance in Case 1 and Case 2. The number and quality of commands given and the child's compliance was scored using the DPICS-IV during 5 min of PLP in pre- and post-treatment. In both cases, the number of total commands and ineffective commands (commands that were not specific enough or were indirect commands, e.g., suggestions or in question style) had decreased. The percentage of commands that child obeys (showed as compliance (\%) in figure) had increased in both cases. DPICS-IV: Dyadic Parent-Child Interaction Coding System (4th edition), PLP: Parent-Led Play 


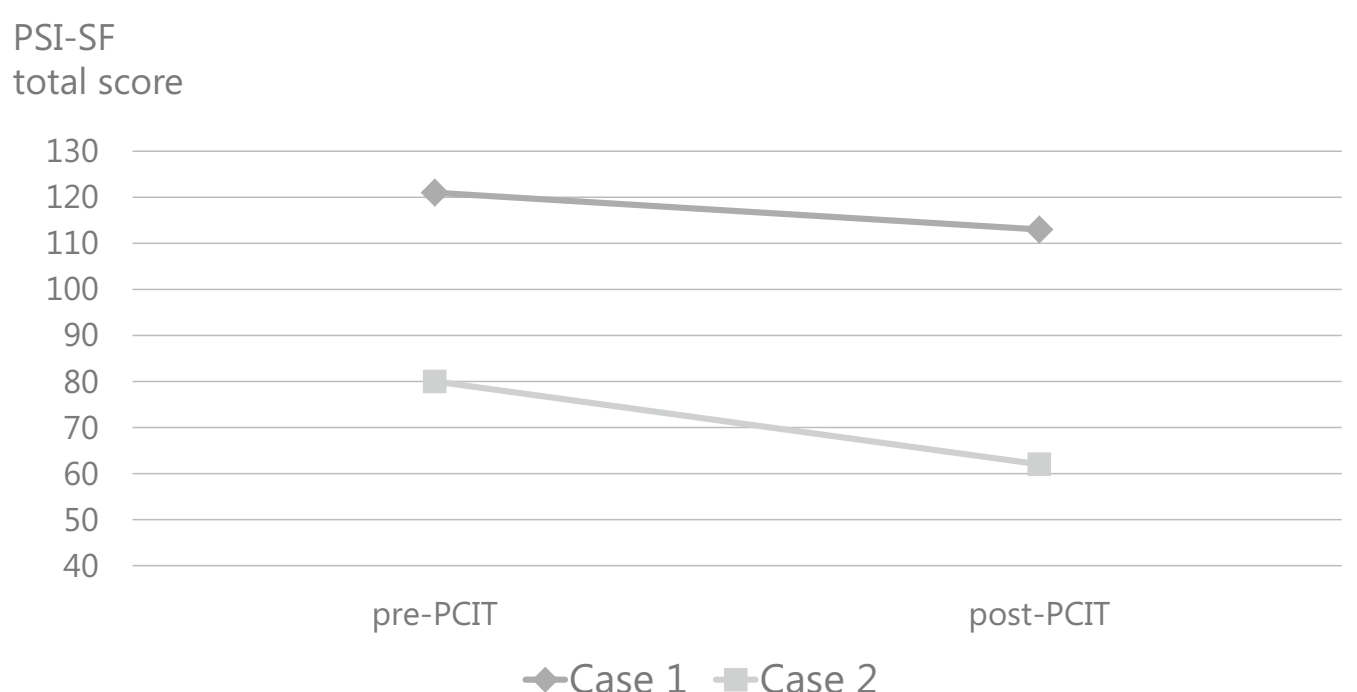

Fig. 4 Changes in Parenting Stress Scores according to PSI-SF in Case 1 and Case 2. The total scores of the PSI-SF in pre- and post-treatment are shown. Both cases showed reduction in total scores. In PSI-SF, high scores show high parenting stress. PSI-SF: Parenting Stress Index-Short Form

was often scolded by his teachers because he was unable to follow instructions despite of his high comprehension. After psychiatric evaluation, Kou was diagnosed as Attention-Deficit Hyperactivity Disorder (ADHD)-Combined Type. His psychiatrist performed an interview and observation about the ADHD symptoms according to the DSM-5 diagnostic criteria [11]. The presence of impairment was observable both at home and at pre-school. He did not fulfill the diagnostic criteria for Autism Spectrum Disorder. Medication was not recommended by his psychiatrist, and weekly PCIT was recommended for Kou and Ms. Kawa. PCIT sessions were held by two PCIT therapists using the PCIT 2011 protocol when he was 4 years and 3 months old.

At baseline, his ECBI scores were 126 on the intensity scale and 8 on the problem scale. Baseline interactions revealed that Kou was a very active and inquisitive boy and was very talkative during play. When he was stacking blocks high and was not able to manage the blocks as he desired to, he showed anger and got aggressive. Ms. Kawa was also talkative and asked many questions and made many suggestions during Child-Led Play. It seemed as though she was contributing to Kou's excitability. During Clean-Up, he was able to obey instructions while being very angry. At the end of the session, he had difficulty exiting the play-room and Ms. Kawa tried to encourage him by changing the subject, threatening him and trying to bribe him with snacks; none of this was effective in changing his behaviors. His compliance was $43 \%(=3 / 7)$ during PLP and $67 \%(=2 / 3)$ during CU. His difficulty seemed to lay in controlling his emotions.
During CDI, we tried to strengthen Ms. Kawa's skills by decreasing unnecessary stimuli (i.e., questions and both direct and indirect commands including suggestions) and increasing positive attention using PRIDE skills for appropriate behaviors. Ms. Kawa learned to give praises when he was playing gently and was focusing well, which was the opposite of his problematic behavior at home and at pre-school. Ms. Kawa noticed that Kou was concentrating well when she was continuously giving positive comments to him. She also noticed that when she was busy with her work and went on business trips leaving Kou with his father or grandparents, Kou was not able to sleep well and this had negative influence on his behaviors. She was unable to complete her PCIT homework and sometimes canceled PCIT sessions, so she had difficulty meeting the "mastery criteria." Kou's main psychiatrist had discussed the issue with Ms. Kawa and she tried to schedule her work and gave priority to Kou's schedules and PCIT. She was able to meet "mastery criteria" after 11 sessions of CDI. By this point, his excitability had improved and Ms. Kawa was able to remain calm even when he started to become angry.

During PDI, Ms. Kawa learned to give simple commands and Kou seemed to be willing to comply. $\mathrm{He}$ sometimes missed her commands especially when he was focusing on his play. We asked Ms. Kawa to give a short command to look at her first to seek his attention, praise him for his compliance and then to give the command. This seemed to work well for Kou. Commands were gradually integrated to those used in every-day life and he was able to comply. She also noticed that he was able to comply when she tried to control the frequency and quantity 
of the commands used. She asked the teachers to try to use similar techniques at school (i.e., praising appropriate behaviors and avoiding unnecessary stimuli), and his overall function had improved.

For this case, PCIT consisted of 13 CDI sessions and 10 PDI sessions. Ms. Kawa mentioned that Kou "loved mom" and he understood what would please her. Father and grandparents reported that Kou was able to understand and follow their instructions at once.

Post-treatment evaluation showed that the ECBI scores were 93 on the intensity scale and 1 on the problem scale; both within normal limits (Fig. 1). Ms. Kawa's verbalization showed improvement; she used more statements that promoted positive interaction compared to pretreatment (Fig. 2). Kou's compliance was both $100 \%$ during PLP and CU (Fig. 3). On the PSI-SF, her total stress score decreased from 80 to 62; decrease in stress scores was observed in each domain (Fig. 4).

Follow-up session revealed that his ECBI scores were remained low (103 for intensity scale and 4 for problem scale) and his behavior at school was well maintained (Fig. 4).

\section{Discussion}

Two cases with ADHD were illustrated. PCIT was effective in both cases with improvement in problematic behaviors. Although hyperactive symptoms remained in both cases, mothers in both cases showed reduction in stress scores and felt confident in taking charge of their children.

From the behavioral perspective, PCIT strengthens appropriate behaviors by giving positive reinforcement using PRIDE skills while withholding attention to inappropriate behavior expecting for extinction. Also, praising positive behaviors that are opposite of problematic behaviors were effective in increasing adaptive behaviors. For Case 1 (Anna), this corresponded to "speaking with a soft voice" opposed to whining and being noisy; for Case 2 (Kou), this was "playing gently" opposed to restlessness and being over-excited. Giving clear and precise commands and being able to be consistent according to the children's behaviors enabled the children to have clearer images of their expectations. PCIT techniques were generalized to every-day life situations to prevent problematic behaviors outside sessions. Unnecessary stimuli were lowered as possible. For Case 2, the realization of the mother of the link between her business trips and the child's behaviors led her to regulate her work to maximize his performance at pre-school.

From the developmental perspective, children with disruptive behaviors tend to have difficulty building maternal secure attachment. Kissgen showed that maternal insecure attachment was matched with higher ADHD symptoms [16]. During PCIT, the quality of the parentchild interaction improves [17]. Using PRIDE skills for appropriate behaviors enables mothers to increase reflective functioning, which may in turn strengthen the child's self-esteem and promote emotional regulation. Although ADHD is a neuro-developmental disorder with impairments in behaviors and emotional regulation domains, enhancing parenting skills from the developmental perspective seemed to be effective in reducing problematic behaviors.

\section{Conclusion}

According to the two cases reported here, PCIT seems to be effective for Japanese young children with disruptive problematic behaviors. Future studies using randomized controlled trials are recommendable for confirmation.

\section{Abbreviations}

PCIT: Parent-Child Interaction Therapy; ADHD: Attention-Deficit Hyperactivity Disorder; PBT: parent behavior training; Triple P: Positive Parenting Program; CDI: child-directed interaction; PDI: parent-directed interaction; ECBI: Eyberg Child Behavior Inventory; DPICS: Dyadic Parent-Child Interaction Coding System; CLP: Child-Led Play; PLP: Parent-Led Play; CU: Clean-Up; PSI-SF: Parenting Stress Index-Short Form.

\section{Authors' contributions}

$\mathrm{NH}$ was the main therapist for the presented cases and wrote this paper. MK was the main psychiatrist for both cases and recruited them for PCIT. NK collected and analyzed clinical data. KS and TK revised the manuscript critically for important intellectual content. All authors read and approved the final manuscript.

\section{Author details \\ ${ }^{1}$ Department of Child and Adolescent Mental Health, Aiiku Clinic, Maternal and Child Health Center, Imperial Gift Foundation Boshi-Aiiku-Kai, 5-6-8 Minami-Azabu, Minato-ku, Tokyo 106-8580, Japan. ${ }^{2}$ Department of Medi- cal Welfare, Aiiku Clinic, Maternal and Child Health Center, Imperial Gift Foundation Boshi-Aiiku-Kai, 5-6-8 Minami-Azabu, Minato-ku, Tokyo 106-8580, Japan. ${ }^{3}$ Division of Child Welfare and Mental Health, Aiiku Research Institute, Imperial Gift Foundation Boshi-Aiiku-Kai, 5-6-8 Minami-Azabu, Minato-ku, Tokyo 106-8580, Japan. ${ }^{4}$ Wakamatsu-cho Mental and Skin Clinic, 9-4MH Build- ing 1F/B1F, Wakamatsu-cho, Shinjku-ku, Tokyo 162-0056, Japan.}

\section{Acknowledgements}

Not applicable.

\section{Competing interests}

The authors declare that they have no competing interests.

\section{Availability of data and materials}

The datasets used and analyzed during the current study are available from the author on reasonable request.

\section{Consent for publication}

Written informed consent was obtained from the parents of the two patients for publication of this case report. The names used are pseudonyms and all personal information are kept anonymous.

Ethics approval and consent to participate

All study procedures were in accordance with the World Medical Association Helsinki Declaration.

Funding

All authors have no funding to declare. 


\section{Publisher's Note}

Springer Nature remains neutral with regard to jurisdictional claims in published maps and institutional affiliations.

Received: 5 June 2017 Accepted: 14 February 2018

Published online: 24 February 2018

\section{References}

1. Wolraich M, Brown L, Brown RT, Du Paul G, Earls M, Feldman HM, Visser S. ADHD: Clinical Practice Guideline for the diagnosis, evaluation, and treatment of attention-deficit/hyperactivity disorder in children and adolescents. Pediatrics. 2011;128(5):1007-22

2. Gleason MM, Egger HL, Emslie GJ, Greenhill LL, Kowatch RA, Lieberman AF, Zeanah CH. Psychopharmacological treatment for very young children: contexts and guidelines. J Am Acad Child Adolesc Psychiatry. 2007;46(12):1532-72.

3. Charach A, Carson P, Fox S, Ali MU, Beckett J, Lim CG. Interventions for preschool children at high risk for ADHD: a comparative effectiveness review. Pediatrics. 2013;131(5):e1584-604.

4. Barkley RA. Attention deficit hyperactivity disorder. 4th Edition. A Handbook for Diagnosis and Treatment. New York: Guilford Press; 2014

5. Anastopoulos AD, Shelton TL, DuPaul GJ, Guevremont DC. Parent training for attention-deficit hyperactivity disorder: its impact on parent functioning. J Abnorm Child Psychol. 1993;21(5):581-96.

6. Bor W, Sanders MR, Markie-Dadds C. The effects of the Triple P-Positive Parenting Program on preschool children with co-occurring disruptive behavior and attentional/hyperactive difficulties. J Abnorm Child Psychol. 2002;30:571-87.
7. Eyberg SM. Parent-Child Interaction Therapy: integration of traditional and behavioral concerns. Child Fam Behav Ther. 1988;10:33-46.

8. Cooley ME, Veldorale-Griffin A, Petren RE, Mullis AK. Parent-Child Interaction Therapy: a meta-analysis of child behavior outcomes and parent stress. J Fam Soc Work. 2014;17(3):191-208.

9. Hood K, Eyberg SM. Outcomes of parent-child interaction therapy: mothers' reports on maintenance three to six years after treatment. J Clin Child Adolesc Psychol. 2003;32:419-29.

10. Kamo T. Rebuilding of the nurturing relationship and parent-child interaction therapy of mothers and children affected by domestic violence [Article in Japanese]. Seishin Shinkeigaku Zasshi. 2010;112(9):885-9.

11. American Psychiatric Assocation. Diagnostic and statistical manual of mental disorders, 5th edition: DSM-5. Washington DC: American Psychiatric Association; 2013.

12. Eyberg SM, Funderburg B. Kamo T. transl. Japanese Version of ParentChild Interaction Therapy protocol 2011, PCIT International; 2011.

13. Eyberg SM, Pincus D. Eyberg child behavior inventory and Sutter-Eyberg student behavior inventory: Professional manual. Odessa: Psychological Assessment Resources; 1999. (Kamo T. transl. Japanese Version of ECBI, Tokyo, Japan: Chiba Test Center, 2016.).

14. Eyberg SM, et al. Kamo T. transl. Japanese Version of Dyadic Parent-Child Interaction Coding System Clinical Manual (Fourth Edition). PCIT International; 2014.

15. Abidin R. Parenting stress index manual. 3rd ed. Odessa: Psychological Assessment Resources; 1995.

16. Kissgen R, Krischer M, Kummetat V, Spiess R, Schleiffer R, Sevecke K. Attachment representation in mothers of children with attention deficit hyperactivity disorder. Psychopathology. 2009;42:201-20.

17. McNeil C, Hembree-Kigin T. Parent-child interaction therapy. 2nd ed. New York: Springer; 2010.

\section{Submit your next manuscript to BioMed Central and we will help you at every step:}

- We accept pre-submission inquiries

- Our selector tool helps you to find the most relevant journal

- We provide round the clock customer support

- Convenient online submission

- Thorough peer review

- Inclusion in PubMed and all major indexing services

- Maximum visibility for your research

Submit your manuscript at www.biomedcentral.com/submit
() Biomed Central 